\title{
Time evolution of the lateral-velocity distribution for a strong-field-ionization process
}

\author{
I. A. Ivanov* \\ Center for Relativistic Laser Science, Institute for Basic Science, Gwangju 500-712, Republic of Korea \\ and Research School of Physics and Engineering, Australian National University, Canberra, \\ Australian Capital Territory 0200, Australia \\ (Received 29 March 2016; published 3 May 2016)
}

\begin{abstract}
We study time development of a cusp in the lateral-velocity distribution for the process of strong-field ionization. The lateral-velocity distribution is computed using an $a b$ initio quantum mechanical procedure for the moments of time inside and after the end of the laser pulse. We show that at the moment of time corresponding to the midpoint of the laser pulse the lateral-velocity distribution is a smooth Gaussian curve, its parameters agreeing very well with the predictions of the tunelling theories. At the moment of time corresponding to the end of the pulse the lateral-velocity distribution narrows considerably, showing the initial stage of the cusp-formation process due to the Coulomb focusing effect. Following evolution of the ionized wave packet yet further in time we consider the cusp formation in detail.
\end{abstract}

DOI: 10.1103/PhysRevA.93.053403

\section{INTRODUCTION}

The original work by Keldysh [1] and its subsequent developments [2-6], which are sometimes dubbed collectively as tunelling or strong field approximation (SFA) theories, provide a comprehensive foundation for the current understanding of the photoionization process in the tunelling regime [7-9]. This regime is characterized by the small values of the Keldysh parameter $\gamma=\omega \sqrt{2\left|\varepsilon_{0}\right|} / E$ (here $\omega, E$, and $\left|\varepsilon_{0}\right|$ are the frequency, field strength, and ionization potential of the target system expressed in atomic units). A great advantage the tunelling theories, which are typically analytical approaches, possess over the numerical techniques is their ability to provide a clear physical picture of the ionization process. In this picture tunelling photoionization can be described as a process in which an electron emerges into the continuum, undergoing the under-the-barrier tunelling. This event constitutes the first stage of the ionization process and is described quantum mechanically. Tunelling theories provide probabilistic distributions of the electron's characteristics, such as the electron's velocity distributions, at the moment of the electron's exit form under the barrier. Remarkably, once these initial distributions are known, one may successfully reproduce the further stages of the ionization process using simple classical or semiclassical tools. One may use, for instance, these distributions as the initial density distributions in the electron's phase space for the subsequent simulations based on the classical equations of motion [10,11].

Of particular interest is the so-called lateral velocity distribution, which describes the distribution of the electron velocities in the direction perpendicular to the polarization plane of the driving pulse. If we neglect, as is done in many variants of the tunelling theories, the influence of the atomic core on the electron's motion after the ionization event, the lateral velocity distribution should retain its shape in the course of the subsequent evolution. Detector recording this distribution far away from the ionic core would then effectively measure lateral velocity distribution at the moment of the ionization event $[11,12]$.

\footnotetext{
*igor.ivanov@anu.edu.au
}

If the influence of the ionic core on the electron's motion cannot be neglected, the lateral velocity distribution at the moment of the ionization event and the one measured at the detector need not coincide. The tunelling theories predict that lateral velocity distribution at the moment when ionization occurs is typically a Gaussian function of the lateral velocity, with a prefactor which does not play a significant role as long as we are interested in the shape of the distribution $[8,10]$. On the other hand, it is known [13] that for the systems where ionized electrons and the ionic core interact through the Coulomb force, the lateral electron momentum distribution measured at the detector exhibits a cusp at the zero transverse velocity for the case of the linearly polarized driving laser pulse. The physical reason for the appearance of the cusp is the Coulomb focusing effect, i.e., a considerable narrowing of the electron velocity distribution in the directions perpendicular to the laser-field polarization vector due to the presence of the Coulomb field [14]. Mathematically, the origin of the cusp can be traced to the low-energy singularity of the Coulomb wave function [13]. It turns out that an analytic description of the cusp is possible to some extent [15].

The velocity distribution measured at the detector is, in fact, an asymptotic distribution measured at some time in the future when the electron is far away from the ionic core. To compute this distribution one can employ the standard projection procedure [16]. To implement it in practice, the time-dependent Schrödinger equation (TDSE) is propagated in time, and its solution at the end of the laser pulse is projected on the set of the appropriate scattering states of the fieldfree atomic Hamiltonian, viz., the states satisfying the ingoing boundary conditions. If the fieldfree atomic Hamiltonian contains Coulomb interaction, the corresponding scattering states (considered as functions of energy) typically possess low-energy singularities, which naturally leads to a singular behavior of electron velocity distributions, in particular the lateral velocity distribution at zero transverse velocity.

One may adopt, however, a different perspective. The velocity distribution at any given moment of time during or after the end of the pulse is related to the Fourier transform of the TDSE wave function, or, in other words, can be obtained by projecting the TDSE wave function on the set of the plane 
waves (certain care should be exercised depending on the gauge used to describe the atom-field interaction; more on this below). The velocity distributions, obtained by means of the projection on the scattering states of the fieldfree Hamiltonian, are the asymptotic velocity distribution recorded by the detector placed far enough so that electrons reach it long after the end of the laser pulse. Projection of the solution of the TDSE at a given finite moment of time on the set of the plane waves gives us, on the other hand, instantaneous velocity distributions for this particular moment of time. These instantaneous distributions can be used as probability distributions of the initial conditions for the classical electron trajectories [10,11]. According to the tunelling theories, they are smooth Gaussian functions of the velocity components. The emergence of the cusp in the lateral velocity distribution can then be viewed as a time-dependent phenomenon, the cusp appearing as an ultimate result of the evolution of the smooth Gaussian-like distributions in time.

In the present work we will study this process of the time evolution of the lateral velocity distribution in detail. The paper is organized as follows. In Sec. II we describe the numerical techniques we use to perform the TDSE calculation and the procedure we use to compute the lateral velocity distribution. Our results and the conclusions we draw are presented in Secs. III and IV, respectively. Atomic units are used throughout the paper.

\section{THEORY}

\section{A. Solution of the TDSE}

We solve TDSE for a hydrogen atom in the presence of a laser pulse:

$$
i \frac{\partial \Psi(\boldsymbol{r})}{\partial t}=\left[\hat{H}_{\mathrm{atom}}+\hat{H}_{\mathrm{int}}(t)\right] \Psi(\boldsymbol{r}) .
$$

We use the length form for the operator $\hat{H}_{\text {int }}(t)$ describing the interaction of the atom with the laser field:

$$
\hat{H}_{\text {int }}(t)=\boldsymbol{E}(t) \cdot \boldsymbol{r},
$$

The laser pulse is linearly polarized along the $z$ direction, which we use as a quantization axis:

$$
E_{z}=E_{0} f(t) \cos \omega t .
$$

The base frequency of the pulse is $\omega=0.057$ a.u. (wavelength of $790 \mathrm{~nm})$. The pulse envelope function is $f(t)=$ $\sin ^{2}\left(\pi t / T_{1}\right)$, where $T_{1}=3 T$ is the total pulse duration and $T=2 \pi / \omega$ is an optical cycle (o.c.) of the field. We will consider below laser pulses with peak field strengths of $E_{0}=0.0534$ a.u. (intensity of $10^{14} \mathrm{~W} / \mathrm{cm}^{2}$ ) and $E_{0}=0.1 \mathrm{a} . \mathrm{u}$. (intensity of $3.5 \times 10^{14} \mathrm{~W} / \mathrm{cm}^{2}$ ). Electric field of the pulse (3) for the peak field strength of $E_{0}=0.0534$ a.u. is shown in Fig. 1.

The initial state of the system is the ground state of the hydrogen atom. To solve the TDSE we employed the procedure described in Refs. [17-19]. The solution of the TDSE is represented as a series in spherical harmonics:

$$
\Psi(\boldsymbol{r}, t)=\sum_{l=0}^{l_{\max }} f_{l}(r, t) Y_{l 0}(\theta) .
$$

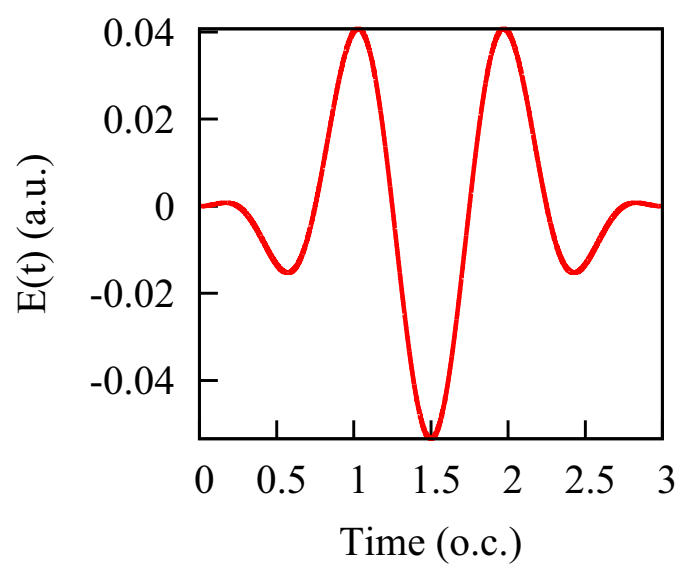

FIG. 1. Electric field of the pulse in Eq. (3) for the intensity of $10^{14} \mathrm{~W} / \mathrm{cm}^{2}$ as a function of time (in units of optical cycles).

The radial variable is treated by discretizing the TDSE on a grid with the step size $\delta r=0.1$ a.u. in a box of the size $R_{\max }$. The values of the parameters $l_{\max }$ and $R_{\max }$ were chosen after the necessary convergence and accuracy checks as $l_{\max }=50$, $R_{\max }=1300$ a.u. for the peak field intensity of $10^{14} \mathrm{~W} / \mathrm{cm}^{2}$, and $l_{\max }=60, R_{\max }=1500$ a.u. for the peak field intensity of $3.5 \times 10^{14} \mathrm{~W} / \mathrm{cm}^{2}$. If we were studying evolution of the system only on the interval of the laser pulse duration, these values of $R_{\max }$ would be excessively large. We will be solving the TDSE, however, on the intervals of time far longer than the pulse duration. Our aim is to follow evolution of the wave packets for several optical cycles after the end of the laser pulse, which necessitates the use of a larger box size.

A system of the coupled equations for the radial functions $f_{l}(r, t)$ resulting after the substitution of the expansion (4) into the TDSE was solved using the matrix iteration method [20]. The time-dependent solution of the TDSE $\Psi(\boldsymbol{r}, t)$ found in this way determines the velocity distribution as a function of time $P(\boldsymbol{v}, t)=|\hat{\Psi}(\boldsymbol{v}, t)|^{2}$, where $\hat{\Psi}(\boldsymbol{v}, t)$ is a Fourier transform of $\Psi(\boldsymbol{r}, t)$.

\section{B. Lateral velocity distribution}

We shall be interested below in the transverse or lateral velocity distributions, describing the probability of detecting an electron with a given value of the velocity component $v_{\perp}$ perpendicular to the laser pulse polarization plane. Because of the symmetry of the problem due to the linear polarization of the driving pulse, any plane containing a polarization vector can be chosen as a polarization plane. Choosing the $(y, z)$ plane as a polarization plane, we obtain for the lateral velocity distribution

$$
P\left(v_{\perp}, t\right)=\int|\hat{\Psi}(\boldsymbol{v}, t)|^{2} d v_{y} d v_{z} .
$$

In the length gauge, which we employ to describe the field-atom interaction, this formula is applicable even for the moments of time inside the laser pulse duration. Had we employed the velocity gauge, the Fourier transform of the velocity gauge wave function $\Psi^{V}(\boldsymbol{r}, t)$ would give us the canonical momentum distribution, which would be different from the velocity distribution because of the well-known 
relation $\boldsymbol{v}=\boldsymbol{k}+\boldsymbol{A}(t)(\boldsymbol{A}(t)$ is the vector potential) between canonical momentum and velocity in the velocity gauge. We would have to use this relation to obtain the velocity distribution from the canonical momentum distribution in the velocity gauge. That would present an additional though not insurmountable technical difficulty, this being the main reason why we choose to use the length gauge in the calculation.

What we need, however, is not the velocity distribution $P\left(v_{\perp}, t\right)$, but the distribution which could be directly compared with the lateral velocity distribution considered in the tunelling theories. To see how and why these two distributions may differ, it is worthwhile to recapitulate briefly the procedure used in the derivations of the velocity distributions in the tunelling theories [8,9,21]. An approach often employed can be conveniently formulated using the Dyson equation for the propagator:

$$
\hat{U}(t, 0)=\hat{U}_{0}(t, 0)-i \int_{0}^{t} \hat{U}(t, \tau) \hat{H}_{\mathrm{int}}(\tau) \hat{U}_{0}(\tau, 0) d \tau,
$$

where $\hat{U}(t, 0)$ is the exact propagator, describing evolution of the atom in the presence of the laser field on the time interval $(0, t), \hat{H}_{\mathrm{int}}(t)$ is the operator of the atom-field interaction, and $\hat{U}_{0}(\tau, 0)$ is the field-free propagator, describing evolution of the atom when laser field is absent. The exact wave function $\Psi(t)=\hat{U}(t, 0) \Psi(0)$, where $\Psi(0)=\phi_{0}$ is an initial ground atomic state with energy $\epsilon_{0}$, is then represented as

$$
\hat{\Psi}(t)=\phi_{0} e^{-i \varepsilon_{0} t}-i \int_{0}^{t} \hat{U}(t, \tau) \hat{H}_{\mathrm{int}}(\tau) e^{-i \varepsilon_{0} \tau} \phi_{0} d \tau .
$$

The second term on the right-hand side of the Eq. (7) describes ionized electrons, which eventually reach the detector. It is this term which is used to derive velocity distribution in the tunelling approaches, typically by obtaining saddlepoint estimates for the integral. The velocity distribution defined in Eq. (5) describes the velocity distribution for all electrons, both ionized and bound. It is, of course, a perfectly acceptable distribution, since electrons remaining bound also contribute to the total velocity distribution. This is not, however, the distribution recorded by the detector placed far from the ionic core, which can measure only the contribution of the ionized electrons. To be able to make a direct comparison with the distribution measured by the detector and given by the tunelling theories, we have to subtract the bound electrons' contribution. This can be achieved by projecting the bound electrons' contribution out of the TDSE wave function $\Psi(\boldsymbol{r}, t)$, used to define the distribution (5). We introduce, therefore, a distribution $W\left(v_{\perp}, t\right)$ defined similarly to (5):

$$
W\left(v_{\perp}, t\right)=\int\left|\hat{\Psi}^{Q}(\boldsymbol{v}, t)\right|^{2} d v_{y} d v_{z},
$$

where instead of the Fourier transform $\hat{\Psi}(\boldsymbol{v}, t)$ of the TDSE wave function $\Psi(\boldsymbol{r}, t)$, we use the Fourier transform $\hat{\Psi}^{Q}(\boldsymbol{v}, t)$ of the projected wave function $\Psi^{Q}(\boldsymbol{r}, t)=(\hat{I}-\hat{Q}) \Psi(\boldsymbol{r}, t)$, and

$$
\hat{Q}=\sum_{l, \varepsilon_{n}<0}|n l 0\rangle\langle n l 0|
$$

is the projection operator on the bound states of the fieldfree Hamiltonian $\hat{H}_{\text {atom }}$ with all possible $n$ and angular momenta $l$ (due to the conservation of the momentum projection $m$ in the linearly polarized field we have to include only the $m=0$ states).

Based on what we said above, we may conclude that $W\left(v_{\perp}, t\right)$ is the closest counterpart of the velocity distributions provided by the tunelling theories and can be directly compared with them. The distribution $W\left(v_{\perp}, t\right)$ can also be related to the velocity distribution measured by a detector in the asymptotic region (long after the end of the laser pulse and far from the ionic core). A prescription [16], allowing us to obtain ionization probabilities measured by the detector at very large times, consists in projecting the TDSE wave function $\Psi(\boldsymbol{r}, t)$ after the end of the pulse on the set of the scattering states with incoming boundary conditions:

$$
\psi_{v}^{(-)}(\boldsymbol{r})=\sum_{l \mu} i^{l} e^{-i \eta_{l}(v)} Y_{l \mu}^{*}(\boldsymbol{v}) Y_{l \mu}(\boldsymbol{r}) R_{l v}(r) .
$$

For the linearly polarized laser pulse and the coordinate system we employ, only the terms with $\mu=0$ actually contribute to the projection. This projection can be performed at any moment of time after the end of the laser pulse, since the overlap of the TDSE wave function $\Psi(\boldsymbol{r}, t)$ and the function $\psi_{v}^{(-)}(\boldsymbol{r}) e^{-i \frac{v^{2}}{2} t}$ does not depend on time. Dubbing the projection on the Coulomb scattering states (10) the Coulomb transform $\hat{\Psi}_{C}(v, t)$, we obtain the Coulomb projected lateral distribution:

$$
W_{C}\left(v_{\perp}\right)=\int\left|\hat{\Psi}_{C}(\boldsymbol{v}, t)\right|^{2} d v_{y} d v_{z} .
$$

We must have, therefore,

$$
\lim _{t \rightarrow \infty} W\left(v_{\perp}, t\right)=W_{C}\left(v_{\perp}\right) .
$$

For this equation to hold, both plane waves used in the Fourier transform and the Coulomb scattering states used in the Coulomb transform must be normalized in the same way. We use the $\delta\left(\boldsymbol{v}-\boldsymbol{v}^{\prime}\right)$ normalization in both cases. There is no need to perform projection procedure encapsulated in Eqs. (8) and (9) for the case of the Coulomb projection, since Coulomb scattering states and bound states are already orthogonal (barring small numerical inaccuracies).

The properties of the lateral distributions we mentioned above can be summarized in terms of the distributions $W\left(v_{\perp}, t\right)$ and $W_{C}\left(v_{\perp}\right)$ as follows. Lateral distribution $W\left(v_{\perp}, t\right)$, as follows from the tunelling theories, should be, for finite times, a smooth Gaussian function centered at $v_{\perp}=0 ; W_{C}\left(v_{\perp}\right)$ exhibits a cusp at $v_{\perp}=0$. For these properties to be compatible with Eq. (12) we must conclude that the cusp is an asymptotic property, developing in time. The physical origin of the appearance of the cusp is well understood; the cusp is a manifestation of the Coulomb focusing effect [13]. What shall interest us in the next section is how this asymptotic transformation of the Gaussian distribution into the one with a cusp occurs in the course of evolution.

\section{RESULTS}

Using the procedure we described above, we propagate the TDSE on the time interval $\left(0, T_{2}\right)$, where $T_{2}$ is a moment of time larger than the pulse duration $T_{1}$ (which, we note, is 3 optical cycles). Typically we use $T_{2}=10$ optical cycles. The distribution $W\left(v_{\perp}, t\right)$ is computed at various moments 

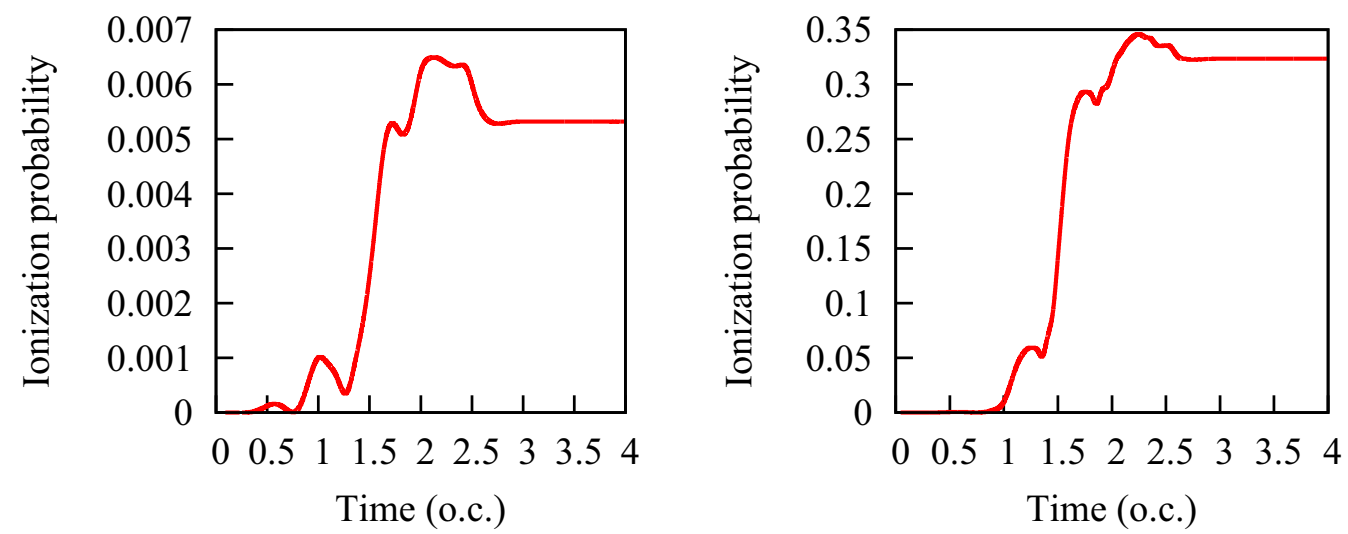

FIG. 2. Left panel: Total ionization probability as a function of time (in units of optical cycles) for the laser pulse described by Eq. (3) with pulse intensity of $10^{14} \mathrm{~W} / \mathrm{cm}^{2}$. Right panel: the same for the field intensity of $3.5 \times 10^{14} \mathrm{~W} / \mathrm{cm}^{2}$.

of time inside and after the end of the interval of the laser pulse duration, using the prescription encapsulated in Eqs. (8) and (9). The wave functions of the bound states were computed by solving the radial Schrödinger equation for the fieldfree hydrogen atom numerically, using the same radial grid we employed for the solution of the TDSE. We used all the bound states with $l \leqslant 15$ to perform the projection in Eq. (9).

As an illustration and an overall consistency check, we present in Fig. 2 results for the total ionization probability as a function of time, defined as an integral $\int_{-\infty}^{+\infty} W\left(v_{\perp}, t\right) d v_{\perp}$. The figure shows expected behavior of the total ionization probability with time, with rapid jumps occurring at the moments of the highest field strength of the laser pulse in Eq. (3), in agreement with the behavior of the instantaneous ionization rate [22]. The probabilities cease to vary after the end of the pulse at the moment $t=3 T$. As we shall see, this is true only for the total ionization probabilities; the lateral distribution $W\left(v_{\perp}, t\right)$ keeps evolving in time long after the end of the laser pulse.

Next, we check that with our definitions of the lateral distributions $W\left(v_{\perp}, t\right)$ and $W_{C}\left(v_{\perp}\right)$ we indeed have the asymptotic condition Eq. (12) satisfied at large times. In Fig. 3 we present results obtained when we use the TDSE solution at the moment $t=10 T$ (seven optical cycles after the end of the pulse). The figure shows results obtained using the nonprojected distribution (5), results obtained using the full projection operation described above (with all negative energy states with $l \leqslant 15$ projected out), results obtained if only the ground state is projected out, and results of the Coulomb projection. The Coulomb projection exhibits a cusp, which is very closely mimicked by the calculation with all bound states with $l \leqslant 15$ projected out. The distribution obtained with only the ground state projected out, however, is only qualitatively correct. It exhibits a cusp but deviates considerably from the results of the Coulomb projection. Nonprojected calculation is both qualitatively and quantitatively incorrect, giving much higher values for the velocity distribution at small velocities. Physical origin of this considerable deviation is clear. The nonprojected calculation, and the calculation with only the ground state projected out, take into account the contributions to the velocity distribution due to the electrons trapped in the bound states after the end of the pulse, which do not have a counterpart in the Coulomb distribution. The contribution of the bound electrons is by far dominating when total ionization probability is small. Using in Eq. (5) the ground-state wave function of the hydrogen atom, one can easily see that the lateral velocity distribution of the electrons in the ground state has a functional form $W_{g}\left(v_{\perp}\right)=A\left(v_{\perp}^{2}+1\right)^{-3}$, with some constant $A$. If we fit the nonprojected lateral velocity distribution using this functional form considering parameter $A$ as a fitting parameter, and
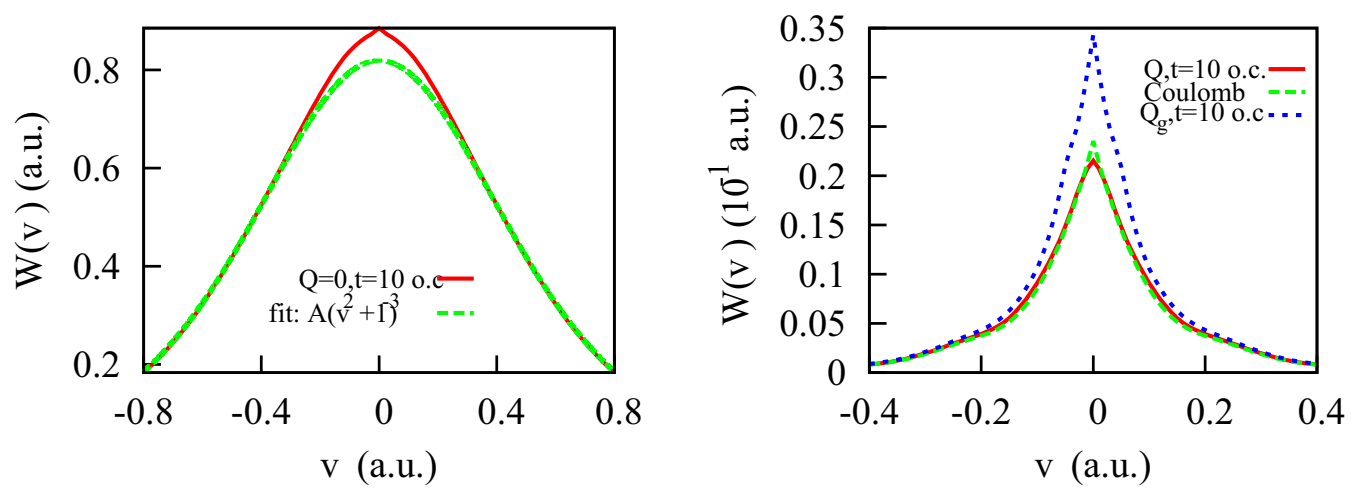

FIG. 3. Lateral distributions computed at the moment $t=10$ o.c. ( 7 optical cycles after the end of the laser pulse) for the peak field strength of 0.0534 a.u. Left panel: Nonprojected distribution (5) is shown as the (red) solid line. Fit based on the equation $A\left(v_{\perp}^{2}+1\right)^{-3}$ is shown as the (green) dash. Right panel: Full projection implemented in Eq. (9): (red) solid line. Only the initial ground state projected out: (blue) dots. Coulomb projection: (green) long dash. 

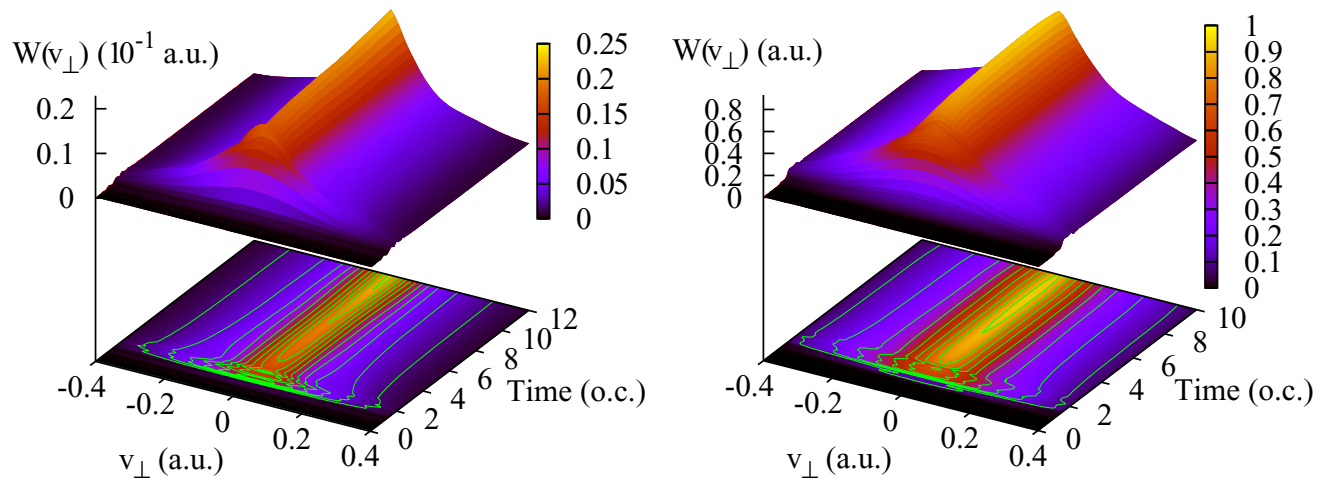

FIG. 4. Evolution of the lateral distributions in time (in units of optical cycles) for the laser pulse (3) with pulse intensity of $10^{14} \mathrm{~W} / \mathrm{cm}^{2}$ (left panel) and $3.5 \times 10^{14} \mathrm{~W} / \mathrm{cm}^{2}$ (right panel).

excluding from the fit the interval $\left|v_{\perp}\right|<0.1$ a.u. of the lateral velocities (where a small cusplike contribution is visible), we obtain the results presented in the left panel of Fig. 3, which show that nonprojected lateral velocity distribution is determined almost solely by the ground-state electrons. As the right panel of Fig. 3 shows, contribution of the excited states to the lateral velocity distribution is also considerable. This illustrates the importance of retaining as many of the bound states in defining the projection operator (9) as possible.

Evolution of the velocity distributions on the intervals of time far longer than the pulse duration is illustrated in Fig. 4, which shows lateral distributions for the intensities of $10^{14}$ $\mathrm{W} / \mathrm{cm}^{2}$ and $3.5 \times 10^{14} \mathrm{~W} / \mathrm{cm}^{2}$. A gradual development of a cusp after the end of the laser pulse is clearly visible in Fig. 4. For the moments of time inside the interval of the laser pulse duration $t \in(0,3 T)$ the cusp has not yet had enough time to develop and the lateral distribution still looks more like a Gaussian function of the transverse velocity. We will consider below both intervals of the time evolution in more detail.

\section{A. Evolution inside interval of the laser pulse duration}

The point we made above about the smooth character of the lateral distribution on the interval of the pulse duration can be better illustrated if we consider a snapshot of the time evolution presented in Fig. 4 for a particular moment of time inside this interval. It is often assumed in numerical simulations [10,11] that electrons are ionized during the small interval around the moment of time when the laser field attains the highest intensity, with the Gaussian velocity distributions (in particular the lateral velocity distribution) predicted by the tunneling theories. The snapshot taken at the moment of time around the midpoint of the pulse would, therefore, be of particular interest. Such snapshots for the moment of time $t=1.5 T$ corresponding to the midpoint of the pulse (3) are shown in Fig. 5 for the laser field intensities of $10^{14} \mathrm{~W} / \mathrm{cm}^{2}$ and $3.5 \times$ $10^{14} \mathrm{~W} / \mathrm{cm}^{2}$.

It is instructive to compare these snapshots with the analytical expressions given by the tunneling theories. An expression for the lateral velocity distribution following from the SFA approach assumes the following form for the hydrogen atom [8]:

$$
W_{\mathrm{SFA}}\left(v_{\perp}\right)=P \exp \left\{-\frac{v_{\perp}^{2} \operatorname{arcsinh} \gamma}{\omega}\right\},
$$

where $\gamma$ is the Keldysh parameter. This expression and its variants (e.g., the limiting form which Eq. (13) assumes for the small values of the Keldysh parameter) are often used in the numerical simulations as a probability measure of different initial conditions for the subsequent classical motion [10]. We shall be using Eq. (13) only with the exponential accuracy, so we shall treat preexponential factor $P$ in Eq. (13) as a constant.

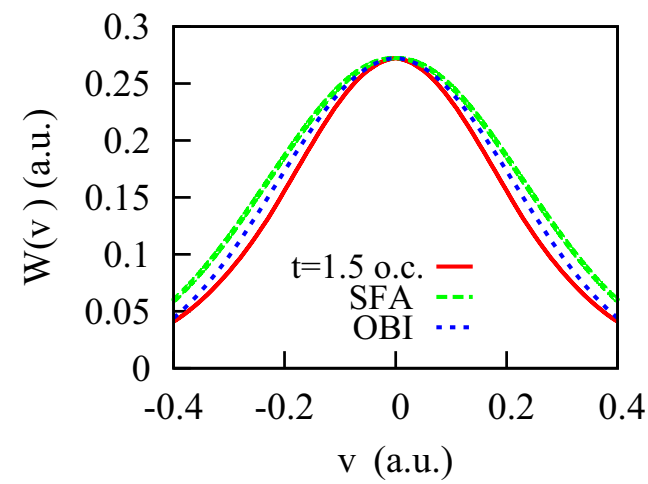

FIG. 5. Lateral velocity distribution at the moment of time $t=1.5 T$ [midpoint of the pulse (3)] for the pulse intensity of $10^{14} \mathrm{~W} / \mathrm{cm}^{2}($ left panel) and $3.5 \times 10^{14} \mathrm{~W} / \mathrm{cm}^{2}$ (right panel). (Red) solid line: calculation based on Eqs. (8) and (9). (Green) dash: SFA approximation (13). (Blue) dots: OBI formula (14). Velocity distributions given by the formulas (13) and (14) have been scaled so that at zero transverse velocities these distributions had the same value as the calculated velocity distribution (8). 
This assumption can be justified on the grounds that for the weak laser fields the effect of the predexponential factor in Eq. (13) on the shape of the velocity distribution can be neglected [10]. Figure 5 shows that exponential dependence (13) very well reproduces the computed distribution (8) for the field intensity of $10^{14} \mathrm{~W} / \mathrm{cm}^{2}$. For the higher field intensity of $3.5 \times$ $10^{14} \mathrm{~W} / \mathrm{cm}^{2}$ we see some deviation of the SFA prediction from the computed distribution (8). This is an anticipated result. For the field strength of 0.1 a.u. we are entering the domain of the over-the-barrier ionization (OBI), where Keldysh theory in its original form is not applicable [9]. The onset of the OBI regime can be estimated from the equation $E_{\text {obi }} \simeq I_{p}^{2} / 2$ (where $I_{p}$ is the ionization potential of the target atom), which follows from considering the Schrödinger equation for the hydrogen atom in an electric field in the parabolic coordinates [23]. An estimate $E_{\mathrm{obi}} \simeq I_{p}^{2} / 4$ following from a simpler picture of the one-dimensional tunelling in the radial coordinate direction can also be found in the literature [24]. These formulas do not account for the above-barrier reflection [24], so they should be considered as order-of-magnitude estimates. For hydrogen atom they place the OBI onset in the interval 0.0625-0.125 a.u. of the values of the laser field strength. Since there is no abrupt transition between the tunelling and the OBI regimes, we may conclude that regardless of the particular formula we use for the estimate of the OBI onset, for the laser field of the order of 0.1 a.u. $\left(3.5 \times 10^{14} \mathrm{~W} / \mathrm{cm}^{2}\right.$ intensity $)$ the OBI effects for hydrogen may well manifest themselves.

For the field strength approaching or exceeding the OBI limit, an analytic formula for the total velocity distribution is available in the literature. For the case of the linearly polarized driving pulse it can be written as follows [24]:

$$
W_{\mathrm{OBI}}(\boldsymbol{v})=\text { const } \times \mathrm{Ai}^{2}\left[\left(2 E_{0}\right)^{-\frac{2}{3}}\left(1+v_{\perp}^{2}+\frac{\omega^{2} v_{\|}^{2}}{3 E_{0}^{2}}\right)\right],
$$

where $\operatorname{Ai}(x)$ is the Airy functionand $v_{\|}$is the velocity component in the direction of the laser polarization vector. This formula reduces to the SFA Gaussian form for weak laser fields, but unlike the velocity distribution given by the SFA, it is expected to be valid for the laser field strength in the OBI regime. Expression (14) gives the distribution for all components of the velocity vector. Lateral velocity distribution can be obtained from the Eq. (14) by integrating over all $v_{\|}$ (this can be done only numerically). The results are shown in the right panel of Fig. 5. The lateral distribution obtained from Eq. (14) is indeed closer to the computed distribution (8) than the one given by the SFA formula (13).

\section{B. Evolution after the end of the laser pulse}

In Fig. 6 we present velocity distributions at the end of the laser pulse $(t=3 T)$ and at the subsequent moments of time. One can see that the distribution $W\left(v_{\perp}, t\right)$ calculated according to Eqs. (8) and (9) at the moment $t=3 T$ still largely retains its Gaussian character, but its width already deviates considerably from the width given by the SFA formula (13). This deviation can be attributed to the initial stages of the Coulomb focusing process, which is already at work.

According to Eq. (12), the distribution $W\left(v_{\perp}, t\right)$ should for $t \rightarrow \infty$ approximate closely the distribution $W_{C}\left(v_{\perp}\right)$ given by Eq. (11), which was obtained by projecting a solution of the TDSE after the end of the pulse (we perform this projection at $t=3 T$ ) on the set of the ingoing hydrogen scattering states (10). One can see that this is indeed the case: The distribution $W\left(v_{\perp}, t\right)$ evaluated at $t=12 T$ for the pulse intensity of $10^{14} \mathrm{~W} / \mathrm{cm}^{2}$ and the distribution $W\left(v_{\perp}, t\right)$ evaluated at $t=$ $10 T$ for the pulse intensity of $3.5 \times 10^{14} \mathrm{~W} / \mathrm{cm}^{2}$ are practically indistinguishable from the corresponding Coulomb projected distributions $W_{C}\left(v_{\perp}\right)$ with cusps at the origin. In Ref. [15] we considered the mathematical origin of the cusp appearance by studying properties of the projection Eq. (11) on the set of the Coulomb scattering states. From that perspective the origin of the cusp is transparent, and the Coulomb wave function is a notoriously singular object (considering its properties as a function of energy for small energies). Overlap of such a singular function and a square-integrable solution of the TDSE can easily produce a singular behavior at zero energy, and it certainly does. The perspective we adopt in the present paper is different. When we project the solution of the TDSE on the set of the plane waves, as we do evaluating $W\left(v_{\perp}, t\right)$ according to Eqs. (8) and (9), we compute essentially overlaps of the wave function defined in Eq. (9), which is a square-integrable
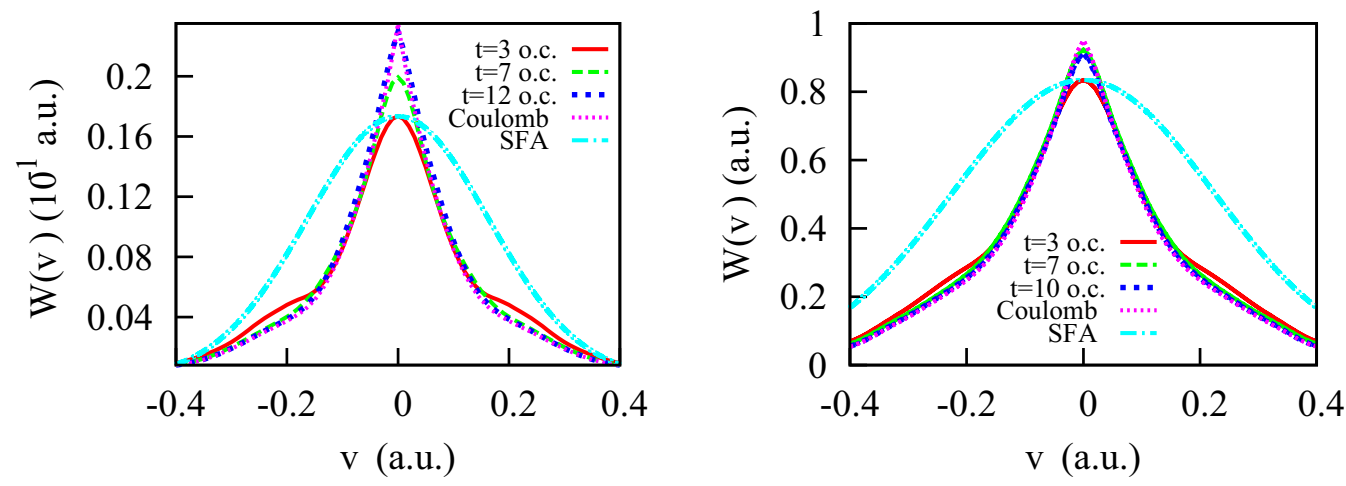

FIG. 6. Left panel: Pulse intensity of $10^{14} \mathrm{~W} / \mathrm{cm}^{2}$. (Red) solid line: $W\left(v_{\perp}, t\right)$ calculated according to Eqs. (8) and (9) at $t=3 T$ (moment of the end of the pulse); (green) long dash: $W\left(v_{\perp}, t\right)$ at $t=7 T$; (blue) short dash: $W\left(v_{\perp}, t\right)$ at $t=12 T$; (magenta) dots: Coulomb projection $W_{C}\left(v_{\perp}\right)$; (cyan) dash-dot: scaled SFA approximation (13). Right panel: Pulse intensity of $3.5 \times 10^{14} \mathrm{~W} / \mathrm{cm}^{2}$. (Red) solid line: $W\left(v_{\perp}, t\right)$ at $t=3 T$; (green) long dash: $W\left(v_{\perp}, t\right)$ at $t=7 T$; (blue) short dash: $W\left(v_{\perp}, t\right)$ at $t=10 T$; (magenta) dots: Coulomb projection $W_{C}\left(v_{\perp}\right)$; (cyan) dash-dot: scaled SFA approximation (13). 
function describing the ionized wave packet, and plane waves $e^{i v \cdot r}$. As is well known, if the square-integrable function decays fast enough with distance, the result of such a projection should be an analytic function of the components of the velocity $\boldsymbol{v}$. The assumption of the fast decay is certainly true in our case, since for any particular moment of time after the end of the laser pulse the spread of the TDSE wave function (8) in the coordinate space remains finite for finite $t$. At first glance, it is not quite clear how an analytic function can approximate the Coulomb projected distribution $W_{C}\left(v_{\perp}\right)$, which has a cusp (and hence a singular point) at the origin. This question can be answered if we take into account the fact that the spread of the TDSE wave packet, though remaining finite at any finite moment of time $t$, increases in the course of the time evolution. As a simple model, illustrating the main features of the cusp appearance viewed from this perspective, we can consider the Fourier transform $\hat{g}(\boldsymbol{v})$ of a square integrable function $g(\boldsymbol{r})$ :

$$
g(\boldsymbol{r})=e^{-\mu(t)(\boldsymbol{r}-\boldsymbol{u} t)^{2}},
$$

where the parameter $\mu(t)$ characterizes the spread of the wave packet in the coordinate space, and $\lim _{t \rightarrow \infty} \mu(t)=0$. Such a wave function describes a wave packet moving with a group velocity $\boldsymbol{u}$ and spreading with time. For the integral of the squared Fourier transform $\hat{g}(\boldsymbol{v})$ integrated over the $v_{y}, v_{z}$ components of the velocity we will get

$$
\int|\hat{g}(\boldsymbol{v})|^{2} d v_{y} d v_{z}=\text { const } \times \exp \left\{-\frac{v_{x}^{2}}{2 \mu(t)}\right\},
$$

where all irrelevant factors, not depending on $v_{x}$, have been absorbed into a constant prefactor. For $\mu \rightarrow 0$ the distribution (16) closely mimics behavior of a function with a cusp at $v_{x}=0$. This simple model illustrates the main idea. If the spread of the wave function in the coordinate space increases with time, one can well expect its Fourier transform integrated over the $v_{y}, v_{z}$ to exhibit singular behavior at $v_{x}=0$ in the limit $t \rightarrow \infty$. The model based on the expression (15) can also be used to illustrate another aspect of the asymptotic relation (12). As we have seen, the spread of the ionized wave packet in the coordinate space accounts for producing the cusplike behavior of the velocity distribution calculated according to Eq. (8). To make the asymptotic equation (12) valid, on the other hand, the distance at which the crest of the wave packet is located ( $u t$ in the case of the simple model above) must grow in time. Suppose that we compute both Fourier and Coulomb transforms of the TDSE wave function defining $W\left(v_{\perp}, t\right)$ and $W_{C}\left(v_{\perp}\right)$ respectively, at some moment of time long after the end of the laser pulse. This recipe of letting the system evolve freely for sufficient time is often used in the photoionization calculations [25], especially in the calculations performed for the systems for which continuous spectrum states are difficult to calculate. The main idea of the recipe is that if the system is allowed to evolve freely for long enough time, then only the asymptotic form of the continuous spectrum wave function on which we project really matters for the calculation of the projection integral. Calculating the Coulomb and Fourier transform of the TDSE wave function we may use then the asymptotic form of the Coulomb scattering wave function. The latter is given by the well-known formula [23]: $\Psi_{v}^{-} \propto e^{i v \cdot r+i \gamma(\boldsymbol{r}, \boldsymbol{v})}$, where the function $\gamma(\boldsymbol{r}, \boldsymbol{v})$ depends on $\boldsymbol{r}$ logarithmically. The spatial derivatives of this factor then decay as $r^{-1}$ for large $r$. Therefore, for large enough times, when the bulk of the ionized wave packet is at large distances from the core, this factor can be considered as essentially constant, contributing only an unimportant constant phase factor to the overlap integral.

\section{CONCLUSION}

We studied the process of the cusp formation in the lateral velocity distribution in time. We adopted the definition of the lateral velocity distribution based on Eq. (8) and the projection procedure (9). The projection procedure was needed to exclude the contributions of the bound electrons (which is perfectly physical, but did not interest us) and thereby to enable direct comparison with the SFA predictions for the lateral velocity distribution for the ionized wave packet.

Such a comparison shows that at the moment of time corresponding to the midpoint of the laser pulse (3) (the highest instantaneous laser intensity), which in SFA is interpreted as a moment when ionization event occurs, the lateral velocity distribution is a smooth Gaussian-like curve. The width of this curve, furthermore, agrees very well with the SFA prediction (13) for the peak laser field intensity of $10^{14}$ $\mathrm{W} / \mathrm{cm}^{2}$, and to a lesser degree, but still quite satisfactorily, for the peak laser field intensity of $3.5 \times 10^{14} \mathrm{~W} / \mathrm{cm}^{2}$. In the latter case, the agreement can be improved if one takes into account that the field intensity of $3.5 \times 10^{14} \mathrm{~W} / \mathrm{cm}^{2}$ borders the onset of the over-the-barrier ionization regime for hydrogen atom, where the Gaussian form of the lateral velocity distribution is to be replaced with the one following from the more general expression (14), which is expected to be valid in the OBI regime. This results show that the assumption often made $[10,11]$ that initial conditions for the classical simulations are distributed according to the SFA formula (13) is indeed very well justified.

We tracked the evolution of the lateral velocity distribution further in time, and saw that at the end of the pulse, while still retaining manifestly Gaussian character, the distribution narrows considerably, which can be attributed to the initial stage of the cusp-formation process due to the Coulomb focusing effect. Following evolution of the ionized wave packet yet further in time, for the moments of time long after the end of the laser pulse, we were able to consider the cusp formation in detail. As we have seen, the snapshots of the lateral-velocity distribution taken several optical cycles after the end of the laser pulse are already virtually indistinguishable from the lateral distribution Eq. (11), obtained by projecting the solution of the TDSE on the set of the ingoing scattering states for the hydrogen atom, in agreement with the asymptotic equality (12). The simple model based on Eqs. (15) and (16) illustrates some mathematical aspects of this transformation of the distribution, which is initially Gaussian, into the distribution possessing a cusp.

\section{ACKNOWLEDGMENT}

This work was supported by the Institute for Basic Science under No. IBS-R012-D1. 
[1] L. V. Keldysh, Sov. Phys. JETP 20, 1307 (1965).

[2] F. H. M. Faisal, J. Phys. B 6, L89 (1973).

[3] H. R. Reiss, Phys. Rev. A 22, 1786 (1980).

[4] A. M. Perelomov, V. S. Popov, and M. V. Terentiev, Sov. Phys. JETP 23, 924 (1966).

[5] M. V. Ammosov, N. B. Delone, and V. P. Krainov, Sov. Phys. JETP 64, 1191 (1986).

[6] O. I. Tolstikhin and T. Morishita, Phys. Rev. A 86, 043417 (2012).

[7] F. Krausz and M. Y. Ivanov, Rev. Mod. Phys. 81, 163 (2009).

[8] V. S. Popov, Phys. Usp. 47, 855 (2004).

[9] S. V. Popruzhenko, J. Phys. B 47, 204001 (2014).

[10] N. I. Shvetsov-Shilovski, D. Dimitrovski, and L. B. Madsen, Phys. Rev. A 85, 023428 (2012).

[11] A. N. Pfeiffer, C. Cirelli, A. S. Landsman, M. Smolarski, D. Dimitrovski, L. B. Madsen, and U. Keller, Phys. Rev. Lett. 109, 083002 (2012).

[12] R. Boge, C. Cirelli, A. S. Landsman, S. Heuser, A. Ludwig, J. Maurer, M. Weger, L. Gallmann, and U. Keller, Phys. Rev. Lett. 111, 103003 (2013).
[13] A. Rudenko, K. Zrost, T. Ergler, A. B. Voitkiv, B. Najjari, V. L. B. de Jesus, B. Feuerstein, C. D. Schröter, R. Moshammer, and J. Ullrich, J. Phys. B 38, L191 (2005).

[14] A. S. Landsman, C. Hofmann, A. N. Pfeiffer, C. Cirelli, and U. Keller, Phys. Rev. Lett. 111, 263001 (2013).

[15] I. A. Ivanov, Phys. Rev. A 92, 063417 (2015).

[16] R. G. Newton, Scattering Theory of Waves and Particles (McGraw-Hill, New York, 1966).

[17] I. A. Ivanov, Phys. Rev. A 90, 013418 (2014).

[18] I. A. Ivanov and A. S. Kheifets, Phys. Rev. A 87, 033407 (2013).

[19] I. A. Ivanov and A. S. Kheifets, Phys. Rev. A 89, 021402 (2014).

[20] M. Nurhuda and F. H. M. Faisal, Phys. Rev. A 60, 3125 (1999).

[21] V. S. Popov, Phys. At. Nucl. 68, 686 (2005).

[22] G. L. Yudin and M. Y. Ivanov, Phys. Rev. A 64, 013409 (2001).

[23] L. D. Landau and E. M. Lifshitz, Quantum Mechanics (Pergamon Press, New York, 1977).

[24] N. B. Delone and V. P. Krainov, Phys. Usp. 41, 469 (1998).

[25] L. B. Madsen, L. A. A. Nikolopoulos, T. K. Kjeldsen, and J. Fernández, Phys. Rev. A 76, 063407 (2007). 